\title{
DESEMPENHO DE COLETORES ANIÔNICOS DE MINERAIS PORTADORES DE FERRO NA CONCENTRAÇÃO DE FELDSPATO POR FLOTAÇÃO
}

\author{
M. F. OLIVEIRA ${ }^{1}$, J. C. SILVA ${ }^{1}$, T. A. MAGALHÃES FILHO ${ }^{1}$, M. G. BERGERMAN ${ }^{2}$ e D. G. HORTA ${ }^{1^{*}}$ \\ ${ }^{1}$ Universidade Federal de Alfenas, Campus de Poços de Caldas \\ ${ }^{2}$ Universidade de São Paulo \\ daniela.horta@unifal-mg.edu.br*
}

Artigo submetido em novembro/2015 e aceito em novembro/2015

DOI: $10.15628 /$ holos.2015.3659

\section{RESUMO}

Feldspato é um termo aplicado a um grupo de aluminosilicatos combinados com proporções variáveis de potássio, sódio, cálcio entre outros cátions. Na indústria de cerâmica, em específico na fabricação de porcelanato de elevada brancura, o teor de óxido de ferro $\left(\mathrm{Fe}_{2} \mathrm{O}_{3}\right)$ deve ser inferior a $1 \%$. Neste contexto, o presente trabalho teve como objetivo reduzir o conteúdo de $\mathrm{Fe}_{2} \mathrm{O}_{3}$ do minério rico em feldspato (foiaíto), do maciço de Poços de Caldas por meio da técnica de flotação reversa, para atender às especificações exigidas pela indústria de cerâmica. Inicialmente a amostra de minério foi preparada com relação a sua granulometria por meio de processos de britagem, moagem e deslamagem. Em seguida, a amostra foi caracterizada por análise de
Fluorescência de Raios-X (FRX) e Difração de Raios-X (DRX). O desempenho de diferentes tipos de coletores aniônicos (ácido graxo, sulfonato, sulfato e hidroxamato) de minerais portadores de ferro em diversas dosagens (200, 400 e $600 \mathrm{~g} / \mathrm{t}$ ) foi avaliado em um estágio de flotação rougher. De acordo com os resultados dos ensaios de concentração, o reagente que apresentou o maior desempenho foi o sulfato MDB908 na dosagem de $600 \mathrm{~g} / \mathrm{t}$. A redução de $\mathrm{Fe}_{2} \mathrm{O}_{3}$ nesta condição foi de 3,1\% para $1,9 \%$. Os resultados indicaram que o teor desejado de $\mathrm{Fe}_{2} \mathrm{O}_{3}$ pode ser alcançado com o aumento da dosagem do reagente selecionado e a introdução de etapas de limpeza.

PALAVRAS-CHAVE: Flotação, feldspato, foiaíto, cerâmica.

\section{PERFORMANCE OF IRON-BEARING MINERALS ANIÔNIC COLLECTORS IN THE FELDSPAR CONCENTRATION BY FLOTATION}

\begin{abstract}
Feldspar is a term applied to a complex of aluminum silicate which is combined with diverse proportions of potassium, sodium, calcium and other cations. In the ceramics industry, specifically in high whiteness porcelain manufacturing, the percentage of iron oxide $\left(\mathrm{Fe}_{2} \mathrm{O}_{3}\right)$ must be less than $1 \%$. In this context, the objective of the present work was to reduce the $\mathrm{Fe}_{2} \mathrm{O}_{3}$ content of a feldspar ore (foiaite), from the Poços de Caldas massif by means of reverse froth flotation in order to meet the ceramics industry specifications. The performance of different types of iron-bearing minerals anionic collectors (fatty acid, sulfate, sulfonate and hydroxamate) under
\end{abstract}

diverse dosages $(200,400$ and $600 \mathrm{~g} / \mathrm{t}$ ) was evaluated by rougher froth flotation. Initially, the ore sample was prepared regarding its particle size by crushing, grinding and classifying. Then, the sample was characterized by Xray Fluorescence (XRF) and X-ray Diffractrometry (DRX). According to the concentration results, the reagent which presented the highest performance was the MDB908 sulfate at a dosage of $600 \mathrm{~g} / \mathrm{t}$. In this condition, the content of $\mathrm{Fe}_{2} \mathrm{O}_{3}$ decreased from $3.1 \%$ to $1.9 \%$. The results indicated that the intended $\mathrm{Fe}_{2} \mathrm{O}_{3}$ content could be achieved by increasing the selected reagent dosage and introducing cleaning steps.

KEYWORDS: Froth flotation, feldspar, foiaite, ceramic. 


\section{INTRODUÇÃO}

O minério rico em feldspato se caracteriza por sua fusibilidade. Por isso, as indústrias de cerâmica e vidro são as principais consumidoras desse minério (BRAGA et. al, 1998). No Brasil a reserva medida de feldspatos é da ordem de 316,6 milhões de toneladas, distribuída entre os estados do Paraná (28,3\%), Minas Gerais (13,3\%), Paraíba (10,4\%), Rio Grande do Norte $(10,2 \%)$, Rio de Janeiro (10,2\%), Bahia (8,9\%), São Paulo (8,2\%), Santa Catarina $(6,2 \%)$ e $4,3 \%$ em outros estados (PEREIRA, 2013).

Com a expansão da indústria de cerâmica e vidro, aumentou também a demanda de minerais feldspáticos que satisfazem as normas de exigência desse mercado. Não é comum ou necessário que o beneficiamento desta rocha compreenda etapas de concentração. No entanto, a redução de minérios de alto teor tem como consequência a necessidade de utilização de rochas com maior quantidade de impurezas. A presença de ferro, por exemplo, reduz a brancura de peças cerâmicas. Neste contexto, surge a necessidade de investigação de aplicação de métodos de concentração para adequar a composição da rocha potássica destinada a produção de cerâmica (AGUS, et al., 2000; ANDRADE et al., 2005 e BAYAT et al., 2006).

A utilização de flotação isolada ou combinada com a separação magnética são técnicas de beneficiamento que reduzem significativamente a concentração de materiais máficos de alguns minerais como albita, pegmatita e resíduos graníticos de pedreiras (AGUS et. al, 2000). A flotação seguida de separação magnética forma uma combinação de grande eficiência no tratamento de resíduos de pedreiras (ANDRADE et al., 2005 e BAYAT et al., 2006).

O maciço de Poços de Caldas é constituído por rochas ricas em feldspato (nefelina sienitos) e chamadas, de acordo com o ponto de vista geológico, de foiaítos (plutônicas) e tinguaítos (subvulcânicas) (ULBRICH,1993). A rocha potássica de Poços de Caldas apresenta teores de óxido de ferro de 2 a $6 \%$, o que a torna semelhante aos feldspatos de outras partes do Brasil, como São Paulo e Santa Catarina. Assim, torna-se necessário a concentração de feldspato para a remoção de ferro $\left(\mathrm{Fe}_{2} \mathrm{O}_{3}<1 \%\right)$ com o objetivo de permitir sua aplicabilidade na indústria de vidro e cerâmica (BRAGA et al., 1998).

Na Turquia, a separação entre feldspatos e silicatos é realizada por meio de flotação reversa de mica e silicatos em que primeiramente a mica é flotada utilizando amina como agente coletor em $\mathrm{pH}$ entre 2,5 e 3,5. Posteriormente, flota-se silicatos portadores de ferro com coletores do tipo sulfonatos em pH 3-4 (EL SALMAWY et al., 1993). A flotação reversa de silicatos com coletor aniônico do tipo sulfonato também pode ocorrer em solução alcalina, entre pH 10-11, na presença de íons metálicos como $\mathrm{Ca}^{+2} \mathrm{Ba}^{+2} \mathrm{e} \mathrm{Sr}^{+2}$. Tais íons ativam, seletivamente, a superfície dos silicatos, por meio da formação de hidroxocomplexos positivamente carregados (EL SALMAWY et al., 1993). Agus et. al (2000) obteve como resultado do tratamento de minério rico em albita, aliminosilicato de sódio pertencente à família dos feldspatos plagioclásios, uma redução de $88,46 \%$ da concentração de $\mathrm{Fe}_{3} \mathrm{O}_{2}$. A concentração foi conduzida por flotação reversa, utilizando uma dosagem de $500 \mathrm{~g} / \mathrm{t}$ de éster fosfórico em polpas ácidas.

No Brasil, os pegmatitos são a principal fonte de feldspato. Uma rota de beneficiamento por flotação foi desenvolvida pelo Centro de Tecnologia Mineral (CETEM). A estratégia consiste na flotação de mica com reagente catiônico em pH 1,5, seguida da flotação da muscovita com 
reagente aniônico (sulfonato de petróleo) em pH 3,0 (ANDRADE ET AL., 2004). Azevedo Filho (2013) investigou a concentração do feldspato brasileiro vindo da região de Seridó no Rio Grande do Norte. $\mathrm{O}$ trabalho consistiu na comparação entre as rotas direta e reversa de flotação e análise da influência do pH (7 e 11) utilizando-se oleato de sódio como agente coletor. Os resultados obtidos indicaram que a flotação reversa utilizando $\mathrm{pH} 7$ foi a que apresentou maior desempenho (AZEVEDO FILHO, 2013).

O objetivo deste trabalho é reduzir o conteúdo de $\mathrm{Fe}_{2} \mathrm{O}_{3}$ do foiaíto de Poços de Caldas para valor inferior a $1 \%$, visando sua aplicação na indústria de cerâmica (fabricação de porcelanato de elevada brancura).

\section{METODOLOGIA}

\subsection{Preparação da amostra}

O minério de feldspato (foiaíto) proveniente do maciço de Poços de Caldas, com granulometria inferior a $105 \mu \mathrm{m}$, foi fornecida pela empresa Mineração Curimbaba. O minério foi deslamado por sifonação, em que cada amostra de $2 \mathrm{Kg}$ foi misturada com água a uma porcentagem de sólidos de $25 \%$. Em seguida, o pH foi elevado a 10,5 com a utilização de solução de $10 \%$ de hidróxido de sódio $(\mathrm{NaOH})$. O tempo de decantação foi ajustado para que $90 \%$ das partículas removidas na sifonação tivessem tamanho inferior a $44 \mu \mathrm{m}$. O tempo que atendeu a esse requisito foi de 3 minutos. Após a deslamagem, as amostras foram secas em estufa e agrupadas em pilha longitudinal de onde foram retirados padrões de $500 \mathrm{~g}$, que foram empregadas nos ensaios de flotação.

\subsection{Caracterização}

A composição química da amostra de alimentação da flotação foi analisada por Fluorescência de raios-X (FRX) para a determinação dos principais óxidos presentes: $\mathrm{SiO}_{2}, \mathrm{TiO}_{2}$, $\mathrm{Fe}_{2} \mathrm{O}_{3}, \mathrm{~K}_{2} \mathrm{O}, \mathrm{Al}_{2} \mathrm{O}_{3}, \mathrm{CaO}, \mathrm{P}_{2} \mathrm{O}_{5}, \mathrm{MnO}, \mathrm{ZrO}_{2}, \mathrm{MgO}, \mathrm{Na}_{2} \mathrm{O}, \mathrm{Cr}_{2} \mathrm{O}_{3}$. A amostra também foi analisada quanto a sua perda ao fogo (PF) conduzida por calcinação em mufla. Além disso, a amostra foi submetida a análise de Difração de raios-X (DRX) para a determinação da composição mineralógica.

\subsection{Ensaios de flotação}

Os ensaios de concentração por flotação reversa de minerais portadores de ferro foram realizados em célula mecânica de bancada (Engendrar, modelo CFB 1000N). Primeiramente, o pH da polpa que se encontrava próximo de 10 foi ajustado para aproximadamente 4 com solução de ácido sulfúrico $\left(\mathrm{H}_{2} \mathrm{SO}_{4}\right)$ a $10 \%$. Em seguida, o coletor de minerais portadores de ferro foi dosado e condicionado por 4 minutos. Após 2 minutos de condicionamento o espumante óleo de pinho foi dosado $(147 \mathrm{~g} / \mathrm{t}$ ). A flotação rougher foi conduzida durante 5 minutos. Os produtos flotado e afundado foram secos em estufa a $90^{\circ} \mathrm{C}$ e suas massas foram medidas e enviadas à análise química (FRX).

Foram realizados 15 ensaios de flotação utilizando 5 diferentes tipos de coletores aniônicos de minerais portadores de ferro, nas dosagens de 200, 400 e $600 \mathrm{~g} / \mathrm{t}$. Os reagentes testados foram: 
RADIACID 0166 (ácido graxo) da Oleon, MDB 1425 (sulfonato) e MDB 908 (sulfato) da AkzoNobel, SYLFAT FA-1 SPECIAL (SFS) (ácido graxo) da Arizona Chemical e AERO 6493 (hidroxamato) da Cytec.

\section{RESULTADOS E DISCUSSÃO}

A análise de FRX revelou a presença de $3,1 \%$ de $\mathrm{Fe}_{2} \mathrm{O}_{3}$ (Tabela 1). Além disso, a presença de

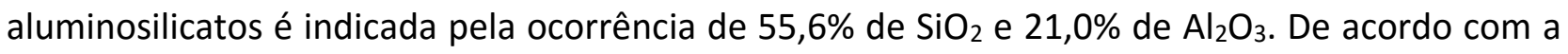
análise de DRX a amostra é composta pelos seguintes minerais: ortoclásio ( $\left.\mathrm{KAISi}_{3} \mathrm{O}_{8}\right)$, nefelina $\left(\mathrm{KNa}_{3}\left(\mathrm{AlSiO}_{4}\right)\right)$, analcima $\left(\mathrm{Na}\left(\mathrm{AlSi}_{2} \mathrm{O}_{6}\right)\left(\mathrm{H}_{2} \mathrm{O}\right)\right)$, feldspato potássico $\left(\mathrm{K}\left(\mathrm{AlSi}_{3} \mathrm{O}_{8}\right)\right)$, labradorita $\left((\mathrm{Ca}, \mathrm{Na})(\mathrm{Si}, \mathrm{Al})_{4} \mathrm{O}_{8}\right)$, egirina $\left((\mathrm{Na}, \mathrm{Ca}) \mathrm{Fe}^{+3} \mathrm{Si}_{2} \mathrm{O}_{6}\right)$, sodalita $\left(\mathrm{Na}_{8}\left(\mathrm{AlSiO}_{4}\right)_{6} \mathrm{Cl}_{2}\right)$, forsterita $\left(\mathrm{Mg}_{2} \mathrm{Si}_{5} \mathrm{Al}_{4} \mathrm{O}\right)$. A presença de feldspatos de sódio e potássio é confirmada pelo conteúdo de $\mathrm{K}_{2} \mathrm{O}(8,5 \%)$ e $\mathrm{Na}_{2} \mathrm{O}$ $(8,3 \%)$ da análise de FRX. De acordo com a análise de DRX o ferro parece estar presente na forma de silicato $(\mathrm{Na}, \mathrm{Ca}) \mathrm{Fe}^{+3} \mathrm{Si}_{2} \mathrm{O}_{6}$.

Tabela 1. Análise de FRX da alimentação da flotação

\begin{tabular}{|c|c|}
\hline Óxido do Elemento & Porcentagem \\
\hline $\mathrm{SiO}_{2}$ & 55,6 \\
\hline $\mathrm{TiO}_{2}$ & 0,4 \\
\hline $\mathrm{Fe}_{2} \mathrm{O}_{3}$ & 3,1 \\
\hline $\mathrm{K}_{2} \mathrm{O}$ & 8,5 \\
\hline $\mathrm{Al}_{2} \mathrm{O}_{3}$ & 21,0 \\
\hline $\mathrm{CaO}$ & 0,9 \\
\hline $\mathrm{P}_{2} \mathrm{O}_{5}$ & 0,0 \\
\hline $\mathrm{MnO}$ & 0,2 \\
\hline $\mathrm{ZrO}_{2}$ & 0,1 \\
\hline $\mathrm{MgO}$ & 0,2 \\
\hline $\mathrm{Na}_{2} \mathrm{O}$ & 8,3 \\
\hline $\mathrm{Cr}_{2} \mathrm{O}_{3}$ & 0,0 \\
\hline Perda ao fogo & 1,5 \\
\hline
\end{tabular}

$\mathrm{Na}$ Figura 1 está ilustrada a relação de $\mathrm{Fe}_{2} \mathrm{O}_{3}$ e recuperação em massa no concentrado com a dosagem de coletor para os diversos reagentes utilizados. Observa-se que a diferença de teor de $\mathrm{Fe}_{2} \mathrm{O}_{3}$ obtido com os diferentes reagentes é significativa apenas em dosagens superiores a $200 \mathrm{~g} / \mathrm{t}$. Além disso, não houve redução de teor de $\mathrm{Fe}_{2} \mathrm{O}_{3}$ em relação à alimentação nesta dosagem, para nenhum dos reagentes experimentados. 


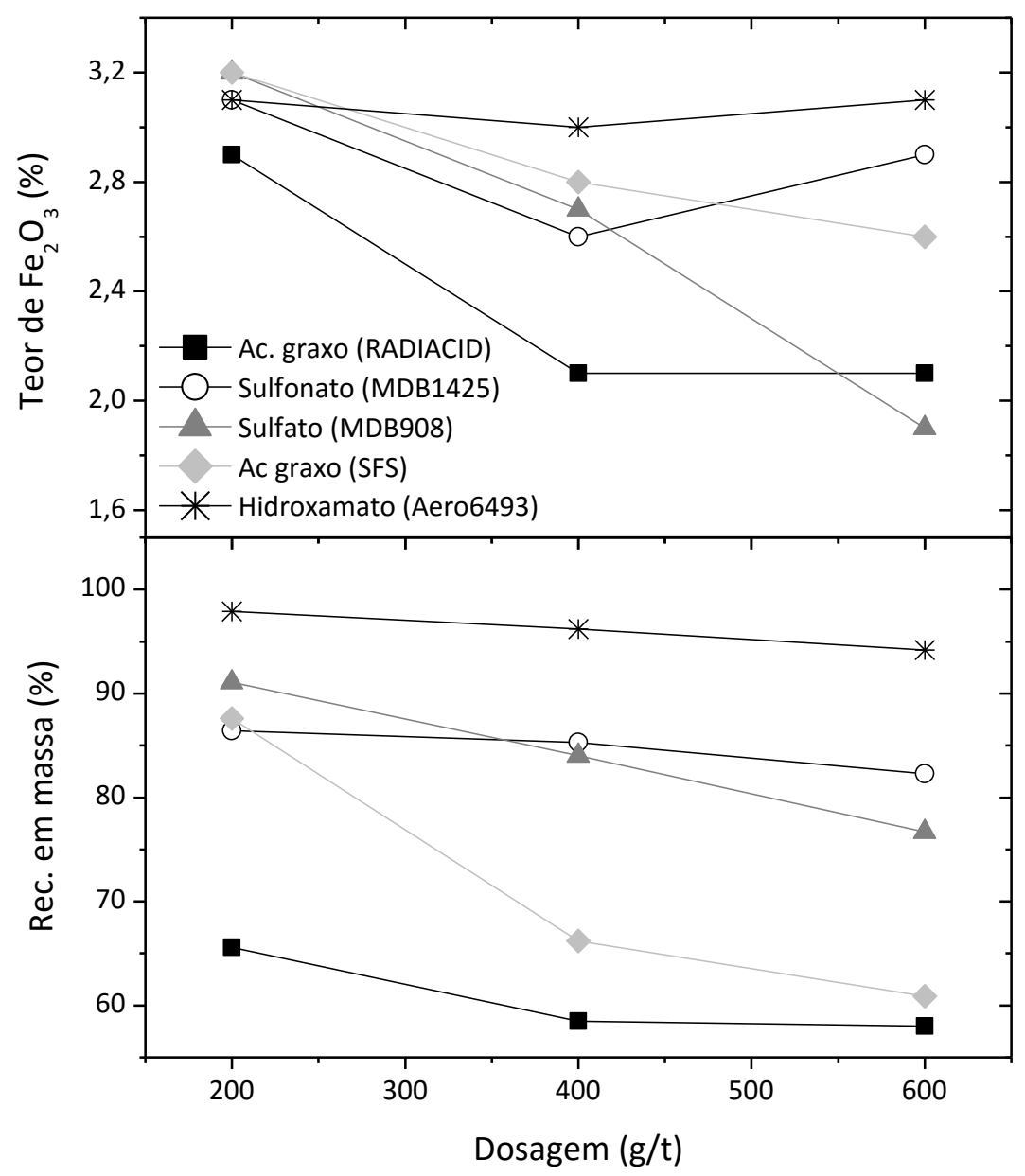

Figura 1. Variação de teor de $\mathrm{Fe}_{2} \mathrm{O}_{3}$ com a dosagem para todos os coletores aniônicos utilizados.

O coletor hidroxamato não é adequado para a remoção dos minerais portadores de ferro contidos na amostra, uma vez que não houve redução significativa do teor de $\mathrm{Fe}_{2} \mathrm{O}_{3}$ (Figura 1). Este comportamento é evidente em todas as dosagens testadas e pode ser explicado pelo fato de o hidroxamato apresentar maior efetividade na flotabilidade da hematita na região de $\mathrm{pH}>7,0$ (LOPES, 2009).

Os coletores sulfonato e ácido graxo RADIACID promoveram redução máxima de $\mathrm{Fe}_{2} \mathrm{O}_{3}$ na dosagem de $400 \mathrm{~g} / \mathrm{t}$. Um aumento na dosagem (a partir de $400 \mathrm{~g} / \mathrm{t}$ ) destes reagentes não afeta o desempenho da concentração por flotação. Os teores mínimos obtidos com tais reagentes foram de 2,6\% e 2,1\% para o sulfonato e o ácido graxo RADIACID, respectivamente. No entanto, o ácido graxo RADIACID conduziu a valores inferiores de recuperação em massa (Figura 1).

Por outro lado, a curva de teor versus dosagem para os coletores ácido graxo SFS e sulfato não atingiram o patamar inferior na maior dosagem utilizada $(600 \mathrm{~g} / \mathrm{t})$. Um aumento da dosagem destes reagentes poderia conduzir a redução ainda maior de teor de $\mathrm{Fe}_{2} \mathrm{O}_{3}$. Assim, faz-se necessário testar dosagens superiores a $600 \mathrm{~g} / \mathrm{t}$ para determinar-se a dosagem ideal da flotação rougher. Comparando-se o desempenho dos dois reagentes, na dosagem máxima testada, 0 sulfato promoveu uma redução de $\mathrm{Fe}_{2} \mathrm{O}_{3}$ de $3,1 \%$ para $1,9 \%$ (38,7\% de redução de teor), consideravelmente maior se comparado ao ácido graxo (SFS) que conduziu a uma redução do teor 
de $\mathrm{Fe}_{2} \mathrm{O}_{3}$ para $2,6 \%$ (16,1\% de redução de teor). Novamente, o ácido graxo conduziu a valores de recuperação em massa inferiores.

Com base nos resultados é possível sugerir os próximos passos que deverão ser tomados para otimizar o estudo de concentração de feldspato: Execução de experimentos com o sulfato e maiores dosagens; Introdução de etapas de limpeza; Verificação da adequação de parâmetros hidrodinâmicos como, por exemplo, porcentagem de sólidos; Análise do desempenho da técnica de separação magnética.

\section{CONCLUSÕES}

O coletor aniônico de minerais portadores de ferro que apresentou o maior desempenho na redução do conteúdo de $\mathrm{Fe}_{2} \mathrm{O}_{3}$ foi o sulfato $\mathrm{MBD} 908$ na dosagem de $600 \mathrm{~g} / \mathrm{t}$. $\mathrm{O}$ teor de $\mathrm{Fe}_{2} \mathrm{O}_{3}$ foi reduzido de $3,1 \%$ para $1,9 \%$ (38,7\% de redução de teor). O perfil da curva teor de $\mathrm{Fe}_{2} \mathrm{O}_{3}$ versus dosagem de sulfato MBD908 é decrescente e linear na região de dosagens testada (200-600 g/t), isso indica que o teor de $\mathrm{Fe}_{2} \mathrm{O}_{3}$ deve reduzir ainda mais com um aumento da dosagem. Embora o ácido graxo (RADIACID) tenha sido eficiente na redução do teor de $\mathrm{Fe}_{2} \mathrm{O}_{3}$, a recuperação em massa deste processo foi inferior a $60 \%$.

Apesar do teor de óxido de ferro não ter atingido à especificação da indústria de porcelanato (inferior a $1 \%$ ), este trabalho contribuiu para selecionar o reagente coletor de maior desempenho que deverá ser empregado em ensaios de flotação com etapas de limpeza (cleaner).

\section{REFERÊNCIAS BIBLIOGRÁFICAS}

1. AGUS, M.; ANGIUS, R.; GHIANI, M.; PERETTI, R.; SERCI, A.; ZUCCA A. Beneficiation of low grade feldspar ores for the ceramics industry. In: INTERNATIONAL MINERAL PROCESSING CONGRESS, 21., 2000; Roma. Anais... Roma: XXI International Mineral Processing Congress; vol C. Oral sessions, 2000. p. 11-17.

2. ANDRADE, M. C.; MATOS, T.F.; LUZ, A.B. Aproveitamento de feldspato contido em pegmatitos. In: ENCONTRO NACIONAL DE TRATAMENTO DE MINÉRIOS E METALURGIA EXTRATIVA, 20., 2004. Florianópolis. Anais... Florianópolis: ENTMME; vol. 2, 2004. p. 111-118.

3. ANDRADE, M.C.; SEIDL, P.R.; LUZ, A.B. Obtenção de feldspato a partir de um granito. In: ENCONTRO NACIONAL DE TRATAMENTO DE MINÉRIO E METALURGIA EXTRATIVA, 21., 2005. Natal. Anais... Natal: ENTMME; vol. 2, 2005. p. 446-451.

4. AZEVEDO FILHO, J.B.; SOUZA, R.F.; MELO, J.V.; PAULO, J.B. Avaliação da eficiência na flotação de quartzo e feldspato potássico utilizando planejamento fatorial. In: ENCONTRO NACIONAL DE TRATAMENTO DE MINÉRIO E METALURGIA EXTRATIVA, 25., 2013. Goiânia. Anais... Goiânia: ENTMME; vol. 2, 2013. p. 53-60.

5. BAYAT, O.; ARSLAN, V.; CEBECI, Y. Combined application of different collectors in the flotation concentration of Turkish feldspars. Minerals Engineering, vol.19, p.98-101, 2006.

6. BRAGA, P.F.A.; SAMPAIO, J.A.; LEAL FILHO, L.S. Estudos de Beneficiamento de Feldspato em Rochas Alcalinas. In: ENCONTRO NACIONAL DE TRATAMENTO DE MINÉRIO E METALURGIA EXTRATIVA, 17., 1998. Águas de São Pedro. Anais... Águas de São Pedro: ABM - Associação Brasileira de Metalurgia e Materiais vol.1, 1998. p. 509-519. 
7. EL SALMAWY, M.S.; NAKAHIRO, Y.; WAKAMATSU, T. The role of alkaline earth cations in flotation separation of quartz from feldspar. Minerals Engineering, vol.6, p.1231-1243, 1993.

8. LOPES, G.M. Flotação direta de minério de ferro. 2009. 176 f. Dissertação de Mestrado - Escola de Minas da Universidade Federal de Ouro Preto. Ouro Preto. 2009.

9. MISHRA, S.K. Anionic Colletors in Nonsulfide Mineral Flotation. In: SOMASUNDARAN, P. E MOUDGIL, BM, 1988. New York. Anais... New York: Surfactant Science Series, vol.27, 1988. p.195-217.

10. PEREIRA, J.; FERNANDES, R. Departamento Nacional de Produção Mineral -Sumário Mineral. Feldspato. [Internet] 2013; [citado 2014 Oct 14]. Disponível em: https://sistemas.dnpm.gov. $\mathrm{br} /$ publicacao/mostra_imagem.asp?IDBancoArquivoArquivo $=8984$

11. ULBRICH, M.N.C. Mineralogy of nepheline syenites from the Poços de Caldas alkaline massif SE Brazil: chemistry, X-ray data and microtextures of feldspars. Revista Brasileira de Geociencias, vol. 23, n.4, p. 388-399, 1993. 